(Aus der Universitäts-Nervenklinik in Königsberg [Direktor: Geh.-Rat E. Meyer].)

\title{
Beitrag zur Kasuistik der Erkrankungen mit amyostatischem Symptomenkomplex.
}

\author{
Von \\ Dr. phil. et med. Charlotte Jacob, \\ Assistentin der Klinik. \\ Mit 3 Textabbildungen. \\ (Eingegangen am 12. Dezember 1921.)
}

Alle Versuche einer strengeren Systematisierung der Erkrankungen des lentikulären Systems oder besser der extrapyramidalen Erkrankungen des Nervensystems sind bisher mißlungen. Die Ursache ist zu suchen vor allem in der relativen Seltenheit der beobachteten Fälle, die oft zum Teil erst nach Jahren als in dieses Gebiet gehörig diagnostiziert, bei denen kaum einer in seinen Symptomen, seinem klinischen Verlaufe mit anderen übereinstimmt, bei dem jedesmal neue Erscheinungen, neue Verlaufsarten zutage treten.

Die Zahl der bis jetzt von diesen Erkrankungen ausgeführten Sektionen ist gering, die dabei erhobenen Befunde sind wechselnd und noch nicht zu übersehen. Einen Sektionsbefund von der jetzt unter dem Namen Torsionsdystonie (Torsionsspasmus, Dyst. musc. deform.) ${ }^{1}$ ) bekannten Erkrankung gibt es'meines Wissens bis jetzt noch nicht. Es ist zu erwarten, daß durch die genavere klinische und pathologischanatomische Erforschung der Encephalitis lethargica, die in ihrer weiteren Entwicklung oft den bei Linsenkernerkrankungen ähnliche Zustandsbilder bietet, und die durch ihr gehäuftes Auftreten zu einer Fülle ron Beobachtungen Gelegenheit gibt, auch ei ne bessere Durchforschung und Klärung der eigentlichen Linsenkernerkrankungen möglich wird. Eine genauere Mitteilung aller in dieses Gebiet gehörigen Erkrankungen erscheinen daher vorläufig notwendig.

Es sei mit erlaubt, der Kasuistik 2 in unserer Klinik beobachtete Fälle hinzuzufügen, die bereits im Dezember 1920 von Klieneberger im Verein für wissenschaftliche Heilkunde zu Königsberg vorgestellt worden sind ${ }^{2}$ ).

1) Während der Drucklegung ist eine Arbeit von Cassirer (Klin. Wochenschr. 1. H. 2, 1922) erschienen, in der er Krankengeschichten und Sektionsprotokolle eines Falles mitteilt, bei dem er intra vitam die Diagnose Torsionsspasmus gestellt hat.

$\left.{ }^{2}\right)$ Ref.: Dtsch. med. Wochenschr. 1921, Nr. 8. 
1. F. S., 14 Jahre alt, jüdisch-polnischer Herkunft, kam am 29. VI. 1920 zur Aufnahme in die Klinik. Nach Angabe der Mutter sind ähnliche Erkrankungen in der Aszendenz nicht vorgekommen. Ein jüngerer Bruder des Pat., der dann später auch in der Klinil untersucht werden konnte (vgl. nachstehende Krankengeschichte), leide an einer Verkrümmung des r. Armes. Der Pat. selbst macht über seine Erkrankung folgende Angaben: Als Kind ist er nie krank gewesen, hat keine Kinderkrankheiten durchgemacht. Er besuchte die Volksschule seiner Heimatstadt und hat dort gut gelernt. Seit einem Jahr geht er nicht mehr zur Schule. Die jetzige Erkrankung begann im Sommer 1918. Damals bemerkte Pat., daß sich der r. Fuß nach außen drehte, er bekam dabei Schmerzen im r. Fußgelenk und im Kreuz. Im Winter, ungefähr $1 / 2$ Jahr später, drehte sich der 1. Fuß beim Gehen nach innen; dazu traten Schmerzen im I. Kniegelenk auf, die jedoch nie sehr groß gewesen sind. 5 Monate vor der Aufnahme in die Klinik begann Pat. sich beim Gehen mit dem Körper auf die r. Seite hinüberzulegen, während der Kopf nach 1. hinübergezogen wurde; besonders beim Gehen und Liegen steht das Gesicht ganz nach 1. Keine Schmerzen in der Hals- und Nacken. muskulatur, dagegen seit dem letzten Winter viel Kopfschmerzen. Im Sommer 1919 hatte er zirka 1 Monat lang starkes Nasenbluten, zuerst 3-4 Tage lang ständig, dann 1-2 mal täglich, endlich alle paar Tage einmal. Seit Beginn der Erkrankung bemerkt er eine starke Gewichtsabnahme.

Befund: Allgemeinzustand mäßig, Haut und Schleimhäute mäßig gut durchblutet; angewachsene Ohrläppchen, Andeutung von Tuberc. Darwini. Die inneren Organe o. B. Der Urin frei von Zucker und Eiweiß. Die Pupillen sind mittelweit und rund, $\mathrm{R} / \mathrm{L}+$ prompt $\mathrm{R} / \mathrm{C}$ ebenso, sehr häufiger Lidschlag. Der Augenhintergrund zeigt normale Verhältnisse. Conj.-Refl. fehlt bds., Corn.-Refl. stark herabgesetzt. Fac., Trig. o. B., Rachen- und Gaumenreflexe fehlen. Pat. spricht etwa gebrochen deutsch, sonst ist jedoch die Sprache ungestört. Schreibt orthographisch falsch und etwas ungeschickt, aber durchaus leserlich und nicht zittrig. Refl.: 0. E. 1. + o. B., r. beim Beklopfen der r. Tricepssehne langsame Ulnarflexion der r. Hand. Kn./Phn. r. + , 1. nicht auslösbar (während der Untersuchung starke Spannung der Muskulatur des 1. Oberschenkels). Ach./Ph. +, Pl.Refl. +, Ba. -, Abdom.-Refl. +, Oppenheim -, kein Klonus. Hautschrift vorhanden. Mechanische Muskelerregbarkeit außerordentlich stark erhöht. Motilität: Arme: Keine Spasmen, keine Ataxie, beide Hände stehen fast stets in Supinationsstellung, die Hand im Gelenk stark ulnarwärts gebeugt, r. $>$ l. Beine: Keine Spasmen, keine Ataxie. Muskulatur des 1. Oberschenkels stark kontrahiert. Ständige kleine Zuckungen in der Muskulatur der Beine, vor allem in den Zehen, im 1. Oberschenkel. Im Sitzen: Der ganze Körper, besonders der Oberkörper leicht nach r. übergebeugt und gedreht, l. Schulter hochgezogen, l. Arm näher am Körper als der r., anscheinend zwangsmäßig. Leichte Krümmung der Lendenwirbelsäule nach 1. Der Kopf scheint etwas nach 1. verschoben, das Gesicht wenig nach 1. gedreht. Beim Stehen: Starke Verschiebung des Oberkörpers nach l. unter Hebung der 1., Senkung der r. Schulter. Die r. Hiufte tritt stark hervor, während die l. sich kaum abhebt; Krümmung der Lendenwirbelsäule nach I. stark verstärkt, Kopfhaltung wechselnd, zeitweise gerade. Beim Liegen: Körperhaltung wie beim Stehen, nur weniger ausgeprägt. Liegt hauptsächlich mit der I. Rückenseite auf, Kopf nach 1 . gebeugt und gewendet, das 1 . Bein in toto nach innen, das $r$. nach außen rotiert. Große Zehe des r. Fußes in Beugestellung. Hält beim Gehen das I. Hüftgelenk, das I. Kniegelenk in leicht gebeugter Stellung steif, schleift mit dem 1. Bein etwas am Boden, den r. Fuß nach außen, den 1. nach innen rotiert, die Zehen, besonders des 1. Fußes, krampfhaft gekrümmt. Romberg -; Störungen der Sensibilität finden sich nicht. Wassermann im Blut - Lumbal- 
punktat klar, Druck nicht erhöht, Nonne -, Lymphocyten: 17, Wassermann Elektrische Untersuchung o. B.

Psychisch macht Pat. einen intelligenten, aufgeweckten, fast über seine Jahre hinaus reifen und ernsten Eindruck, gibt kurze und präzise Antworten.

5. VII. 1920. Liegt dauernd in leichter motorischer Unruhe auf der r. Seite, den Oberkörper nach 1. gedreht, den Kopf fast extrem nach 1. gewendet, das Kinn. leicht zur Brust geneigt. R. Knie in einem Winkel von $45^{\circ}$ gebeugt, r. Fuß leicht dorsal flektiert, Zehen in Beugestellung kontrahiert. L. Bein über das r. gedreht, ebenfalls im Kniegelenk, aber weniger stark gebeugt, FuB fast krampfhaft dorsal flektiert, dabei Zehen extrem nach unten gekrampft, so daß der ganze Fuß einen nach unten konvexen Bogen bildet, während im r. Fuß die extrem nach unten gebeugten Zehen im Grundgelenk dorsal flektiert sind.

Der Oberkörper wird fast dauernd bald nach 1 , bald nach r. gedreht, der Kopf hin und her gewendet, die Beine in mäBigem Umfange gebeugt, gestreckt, nach innen und außen rotiert, wobei aber immer die eben beschriebene Stellung bevorzugt wird und stets als dominierend und als Ruhestellung imponiert.

Die Arme werden meist nach oben gelegt, die Unterarme und Hände mit der Volarseite nach oben, Finger gekrümmt und aneinanderliegend, Hand fast extrem in Ulnarstellung. Die Finger werden langsam gespreizt, einander genähert, gebeugt und zuweilen wurmförmig bewegt, während die Zehen und zum Teil auch der $F u B$ in dauernder mehr an Chorea erinnernden Unruhe begriffen sind. Gelegentlich leichtes Grimassieren des Gesichts: Augenaufreißen, Stirnrunzeln, Unruhe der Augen, häufiger Lidschlag.

Die Muskeln der Beine scheinen in dauernder Anspannung; die Arme imponieren mehr als schlaff, während die Muskulatur der Ober- und Unterschenkel und besonders der Zehen vorübergehend krampfbaft gespannt sind. Aufsitzen und Aufstehen mühsam.

19. VII. 1920. Bewegungen im wesentlichen wie früher. Liegt auf dem Untersuchungssofa beide Füße nach r. gedreht, Zehen des r. Fußes im Grundgelenk dorsal flektiert, im 1. Fuß Zehen gekrümmt. Wechselnde Contractur der Schenkelmuskulatur beider Beine. Oberkörper, Kopf nach 1. gedreht. Arme hochgehoben, im Ellbogen gekrümmt. Hände ulnar flektiert, Handflächen nach außen rotiert, Finger gekrümmt. Ständige motorische Unruhe des ganzen Körpers.

23. VII. 1921. Blutbild Hb. 75.

Erythrocyten 4290000 . Leukocyten 5000.

Ausstrich: Polymorphk. $43 \%$. Lymphocyten 53\%. Eosin. 1\%. Üg. Z. 3\%. Nach $100 \mathrm{~g}$ Traubenzucker keine Zuckerausscheidung im Urin.

6. VIII. 1920. Nach einer Probe von $60 \mathrm{~g}$ Lävulose im Urin: Selivanoffsche Probe -.

10. VIII. 1920. Augenblickliche starke Contractur des ganzen 1. Beines, besonders des Oberschenkels, r. Bein schlaff. Bei wiederholten Bewegungen in den schlaffen Gliedern keinen Übergang von Hypotonie zur Hypertonie. Im kontrahierten Bein sind die Reflexe zur Zeit nicht auszulösen, im schlaffen o. B. Noch während der Untersuchung tritt Erschlaffung der vorher kontrahierten Muskelgruppen ein.

Grobe Kraft in den Beinen fast $=0$, in den Armen o." B. ${ }^{\Gamma}$ Bei Auslösen der Armrefexe wird die ulnare Abduction der Hände noch vermehrt. Beim Gehen schiebt sich der ganze Oberkörper nach 1. herüber, die r. Hüfte tritt stark hervor. Skoliose der Lendenwirbelsäule nach 1., Drehung des ganzen oberkörpers nach 1. herum. Das $r^{\top}$ Bein wird im Hiuft- und Kniegelenk vollkommen steif gehalten, das Fußgelenk abduziert, so daß der Fuß mit der ganzen Sohle den Boden berührt. Der 1. Fuß ist leicht im Knie gebeugt, die Zehen in Flexion auf den Boden gepreßt; 
die Hände und Arme stehen weit vom Körper ab. R. Schulter steht bedeutend höher als die 1., hinkt r. stark, macht leicht drehende Bewegungen mit dem Rumpf.

13. bis 18. VIII. In der chirurgischen Klinik zur Anlegung eines Pneumoperitoneums. Ergebnis: „Leber in typischer Weise deformiert, nioht vergrößert und nicht adhärent."

Aus dem Untersuchungsbefund rom 30. II. 1920 entnehme ich folgendes: Keine Starre, kein Grimassieren, Gesichtsausdruck o. B. An den Augen unregelmäßige nystaktische Einstellungszuckungen bei seitlicher Einstellung, auch beim Blick nach oben. Kein Facialisphänomen. Arm- und PatellarRefl. schwer auszulösen wegen der bei der Prüfung in verstärktem Maße auftretenden unwillkürlichen Bewegungen. Wenn es gelingt, die Aufmerksamkeit abzulenken, sind die Achillesreflexe normal auslösbar; zuweilen kommt es dabei nicht zu einem klonusähnlichen Reflex, sondern zu einem Schüttelzittern der Füße. Mechanische Muskelerregbarkeit erhöht, besonders im Biceps deutliche Wulstbildung, die schnell auftritt und ungewöhnlich lange bestehen bleibt. Rohe Kraft gut, keine Ataxie; der l. Fuß hängt dauernd in Spitzfußstellung herab, zuweilen ist der Tibialis stark kontrahiert, so daß man den Eindruck einer Peroneuslähmung gewinnt, auch der r. Fuß steht in Spitzfußstellung. Die Füße, besonders der 1., sind fast dauernd in leichtem Zittern, das unterbrochen wird von vorwiegend athetotischen, seltener choreatischen Bewegungen. Auch sonst leichte Unruhe im Körper, besonders im l. Bein.

Beim Liegen ist die Körperhaltung wie schon oben wiederholt beschrieben. Aufsitzen aus Rückenlage gut, vielleicht etwas grotesk. Auch beim Sitzen Verdrehung des Oberkörpers, starke Skoliose der unteren Brustwirbelsäule nach l., Ausgleichskoliose der Lendenwirbelsäule nach r., extreme Kyphose der Lendenwirbelsäule, dauernder Wechsel der Wirbelsäulenverkrümmung infolge der dauernden Unruhe. Aufstehen aus Sitzen erfolgt schnell, wie er überhaupt allen Bewegungen schnell, fast hastig nachkommt (im Gegensatz zu dem ungeschickten Gang!). Steht, den Oberkörper leicht nach l. und vorn verdreht, das Becken nach r. seitlich und etwas nach hinten vorgestreckt. Stützt sich beim Stehen vorwiegend auf das r. Bein, r. Fuß in Klumpfußstellung. Das 1. Bein wird im Kniegelenk gebeugt gehalten, der laterale Fußrand stark gegen den Boden gepreßt, während der mediale leicht gehoben scheint; die Zehen stehen in extremer fast krampfartiger Plantarflexion. Die Arme hängen schlaff herunter, sind vom Oberkörper abduziert l. $>$ r. Der Kopf ist leicht nach l. gedreht. Unruhe beim Stehen, bedingt durch die Unmöglichkeit, sich auf die FüBe fest zu stützen, die durch dauernde, teils choreatisch, teils athetotisch aussehende Bewegungen der Zehen, zum Teil auch der ganzen Füße, hervorgebracht wird. Bei Besichtigung von hinten fällt die Verdrehung der Wirbelsäule, das Hervorspringen der r. Hüfte noch mehr auf. Die Wirbelsäule erscheint im unteren Brust- und Lendenteil stark nach 1. skoliotisch verkrümmt. Die Verkrümmung wird durch eine rechtsskoliotische Verkrümmung der Oberbrustwirbelsäule nicht ausgeglichen. Eine Lordose oder Kyphose fällt beim Stehen nicht auf.

(Vgl. Bild I u. 2, aus dem nicht nur die eigenartige, hier beschriebene Haltung, sondern auch die Neigung zum Torquieren deutlich ersichtlich ist; man hat den Eindruck, als ob Pat. nicht gerade gehen kann, sondern in charakteristischer Weise torquieren muß.)

Beim Gehen sinkt der Oberkörper ganz nach 1 . herunter, zugleich tritt in der Lendenwirbelsäule eine Kyphose, darüber eine Lordose in die Erscheinung, das 1. Bein wird circumduziert. Mit den Armen werden rudernde Bewegungen ausgeführt, der Oberkörper wird leicht nach l. und r. torquiert. Beide Füße sind 
beim Gehen nach r. seitlich verdreht, der r. Hacken berührt nur gelegentlich den Boden, mit dem l. FuB stampft er fest auf.

Der Gang hat eine deutlich spastische Komponente und wirkt außerdem ausgesprochen grotesk. Er wird ebenfalls gestört durch die choreatischathetotischen Bewegungen der Zehen; dadurch erscheint er sehr unsicher, so,

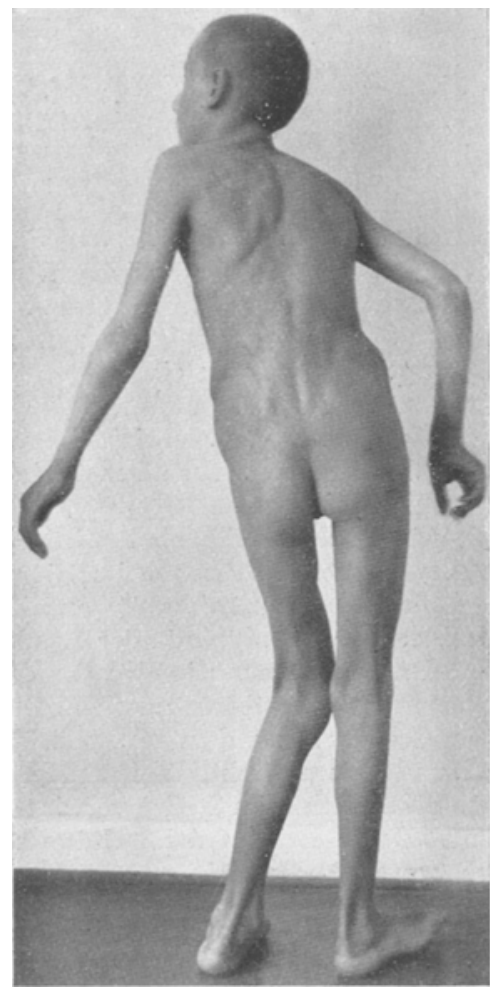

Abb. 1.

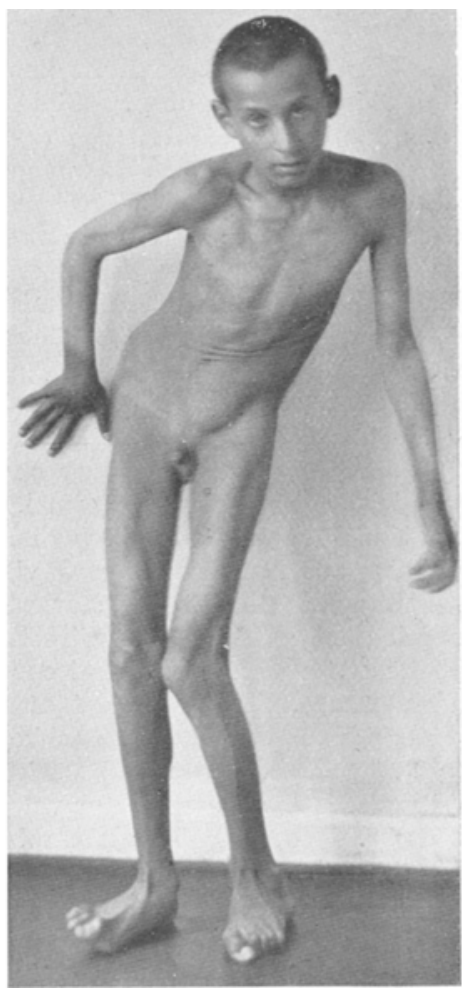

Abb. 2.

als ob Pat. jeden Augenblick Gefahr läuft, zu stürzen. Man hat den Eindruck, daß er, statt zu gehen, mehr von einem Fuß auf den andern stürzt.

4. XII. Urin: E.-Z.-Urobilinogen -.

16. XII. Ein Tropfen Adren. hydrobrom. ins Auge: Keine Reaktion. Injektion von 0,001 Adren. hydrobrom. subcutan: 3 Min. nach der Injektion tritt unter leichtem Erblassen des Pat. eine nur ca. 1 Min. andauernde Erhöhung des Pulsschlages um 12 Schläge ein.

Während der Dauer seines klinischen Aufenthaltes keine Veränderung im Zustand. Keine Klagen über Schmerzen. Ist dauernd guter, gleichmäßiger Stimmung.

Seit seiner Entlassung am 28. XII. 1920 ist nichts mehr über sein Ergehen bekannt.

Da uns der Bruder des Pat. nach seiner Aussage und denen der Mutter auf 
eine ähnliche Erkrankung verdächtig war, so hatten wir ihn auch veranlaßt, die Klinik aufzusuchen. Seine Krankengeschichte ist kurz folgende:

S. S., 13 Jahre alt, als Kind englische Krankheit, sonst stets gesund. Seit Winter 1919 zuerst in der r., etwas später auch in der 1. Hand gelegentlich drehende Bewegungen mit den Fingern. Kann seit Sommer 1920 nicht mehr schreiben. Körperlicher Befund: Seinem Alter entsprechend entwickelt, leichte Asymmetrie des Schädels, des Rumpfes, der Gesichtsbildung, 1. Hüfte steht etwas höher als die r. Augen- Hirnnerven, innere Organe o. B.; im Blut deutliche Lymphocytose $(55 \%)$. Die Sehnenreflexe lebhaft, klonusähnlicher Reflex bei Beklopfen der Achillessehnen (mehr Schütteln und Zittern). Mechanische Muskelerregbarkeit sehr stark erhöht, idiomuskuläre Wulstbildung besonders deutlich im Biceps, die einige Zeit zur Entstehung braucht und dann längere Zeit bestehen bleibt.

Grobe Kraft in den Händen ungewöhnlich gut, ungewöhnlich starkes Vorspringen der Muskelplastik; auch bei häufigem Faustschluß keine Verlangsamung, keine Kraftabnahme; Öfnung stets prompt; feine Fingerbewegungen (Nadelfassen, Zuknöpfen) erschwert. Beine o. B. Gang ohne Störung, Sprache ungestört, Schrift etwas zittrig.

Pat. mußte nach kurzer Beobachtungszeit aus der Klinik entlassen werden, ohne daß uiber die eigentliche Natur der erst im Beginn stehenden und vielleicht auch in das Gebiet der lentikulären Affektionen gehörigen Erkrankung Klarheit errielt werden konnte.

Vergegenwärtigt man sich das Krankheitsbild des ersten Patienten noch einmal, so findet man bei ihm in ausgesprochenstem Maße alle die Symptome vereinigt, die frühere Autoren und zusammenfassend Mendel ${ }^{1}$ ) in seiner Monographie über die Torsionsdystonie als charakteristisch für diese Erkrankung aufgestellt hat. In der seither erschienenen Literatur ist dem wesentlich Neues nicht hinzugefügt. Wir sehen als Hauptkrankheitszeichen einen ständigen Wechsel im Kontraktionszustande einzelner Muskelgruppen, eine andauernde motorische Unruhe von wechselnder Stärke, die durch unwillkürliche Bewegungen von verschiedenstem Charakter (athetoid, choreatisch, ticartig, hemiballistisch) hervorgerufen, ferner die eigenartige, stets in ihrer Stärke wechselnde Deformation der Wirbelsẩule, die sich vollkommen ausgleichen läßt, und der groteske, in seinen einzelnen Phasen kaum zu beschreibende Gang. Charakteristisch ist Zeitpunkt und Art des Beginnes der Erkrankung und die Abstammung des Patienten. Ob die Erkrankung des Bruders der Beginn eines ähnlichen Leidens ist, und dadurch eine familiäre Veranlagung nahelegen würde, muß vorläufig dahingestellt sein.

Der zweite Fall, den ich hier näher beschreiben möchte und der zur selben Zeit in der Klinik beobachtet werden konnte, wurde zu Beginn auch für eine Torsionsdystonie gehalten, bis der weitere Verlauf eine andere Diagnose näher legte.

2. H. F., 12\% Jahre alt, arischer Herkunft, wurde zum ersten Male am 13. IX. 1920 in der Klinik aufgenommen. Aus den anamnestischen Angaben der Pflege-

1) Monatsschr. f. Psychiatr. u. Neurol. 1919, Heft 6; spätere Literatur bei Flater, Zeitschr. f. d. ges. Neurol. u. Psychiatr., Orig. 69. 
mutter und des Hausarztes entnehme ich folgendes: Pat. ist uneheliches Kind, mit 4 Wochen adoptiert; über erbliche Belastung nichts bekannt. War stets schwächlich, entwickelte sich aber normal. Im 6. Lebensjahr machte er einen leichten Scharlach durch. In der Schule kam er schlecht vorwärts. Obgleich, wie die Mutter angibt, sehr rege, machte er auf den Lehrer einen ,recht unfähigen Eindruck, seine Leistungen waren sehr mangelhaft". Die jetzige Erkrankung begann vor ca. 2 Jahren mit Zuokungen in der Gesichtsmuskulatur, Speichellaufen und zunehmender allgemeiner Unsicherheit in den Bewegungen. Eine Entfernung von Rachenwucherungen und roborierende Medikamente führten nur eine vorübergehende Besserung herbei. Vor ca. 1 Jahr stellte sich eine allgemeine körperliche Haltlosigkeit und Schlappheit ein bei stark vermehrtem Speichelfluß und einem eigenartigen kindischen Benehmen. Relativ häufig stürzte Pat. hin, und hat sich dabei im Sommer 1919 zweimal den Arm gebrochen. Auf Veranlassung des Hausarztes war er später mehrere Wochen in Behandlung der hiesigen Kinderklinik. Aus der dortigen Krankengeschichte entnehme ich folgendes: Innere Organe, Sinnesorgane ohne krankhaften Befund. Refl. sehr lebhaft, Ba. - . Der Gang ist etwas steif, täppisch, der Oberkörper wird dabei hin und her gedreht. Bei Beklopfen der Muskulatur entsteht ein Muskelwulst, der peripherwärts wandert. Bewegungen der Hände (Faustschließen und Öffnen) sehr langsam und leicht ungeordnet. Die Bewegungen werden, wenn fortgesetzt, immer langsamer und schwerfälliger. Elektrische Untersuchung: normale Erregbarkeit, faradische und galvanische des Nerv. uln. prompt, kurze Zuckungen. Muskeln faradisch und galvanisch leicht erregbarbar. Bei faradischen Strömen deutlich tonische und nachdavernde Reaktion. Wassermann in Blut und Liquor -.. Psychisch: etwas scheu, geistig nicht ganz vollwertig, kindisches Lachen bei allen Gelegenheiten. Hauptdiagnose: Myasthenie. Nebendiagnose: Imb.

Vor ca. 5 Monaten machten sich im Gegensatz zu der vorher bestehenden Schlappheit und Haltlosigkeit eine eigenartige Starrheit gewisser Muskelgruppen bemerkbar, anfangend im 1. Arm, die sich dann auf die anderen Körperteile verbreitete, seit 4 Wochen besonders auf die Beine.

Bei seiner ersten Aufnahme in die Klinik bot $F$. folgenden körperlichen Befund: zarter Knabe, von ungefähr seinem Alter entsprechender Größe, mit dürftig entwickelter, fast atrophischer Muskulatur, sehr kindlichem Aussehen. Haut und Schleimhäute blaß. Innere Organe ohne krankhaften Befund. Pupillen mittelweit, nicht ganz rund, $\mathrm{R} / \mathrm{L}+, \mathrm{R} / \mathrm{C}+$, Augenbewegungen frei, auch sonst an den Hirnnerven kein krankhafter Befund. Kniephän. lebhaft, andere Refl. von normaler Stärke. Ba. - . Pat. lispelt und spricht leicht verwaschen. Der Mund steht fast ständig offen und sondert reichlich Speichel ab. Die Schrift ist zittrig und undeutlich, eben noch leserlich. In der ganzen Körpermuskulatur macht sich ein deutlicher, ständiger Wechsel von Hypotonie und Hypertonie bemerlsbar, unter besonderer Beteiligung des $x$. Armes, der fast stets stark gespannt erscheint; dabei ständige ausfahrende choreiforme Bewegungen am ganzen Körper. Der sehr eigenartige Gang besteht in kleinen hastigen, tänzelnden Schritten, wobei Pat. die Hacken zuerst aufsetzt; der l. Fuß wird mit dem äußeren Rande aufgesetzt das 1. Bein ist beim Gehen etwas nach innen rotiert, die l. Hand wird gleichzeitig, dabei in eigentümlich extendierter Stellung gehalten, die l. Schulter hochgezogen. Wäbrend des Gehens sind mitunter eigentiimlich schleudernde Bewegungen eines Armes zu bemerken. Eine Lordose besteht nicht, eher eine Kyphose der ganzen Wirbelsäule. Im Liegen lassen die unwillkürlichen Bewegungen nach. Die Sensibilität ist außer einer gewissen Hyperalgesie intakt. Die elektrische Untersuchung zeigt eine gesteigerte galvanische und faradische Erregbarkeit, keine pathologische Reaktion. 
Psychisch benimmt sich Pat. anfangs ruhig und geordnet bei dauernd heiterer Stimmungslage. Allmählich fällt immer mehr ein kindlich läppisches Lachen bei jeder Gelegenheit auf, das wohl schon als zwangsartig und nicht mehr als kindliche Eigenart zu deuten ist.

Pat. wird nach 14 tägiger Beobachtung unverändert nach Hause entlassen. Es wurde damals die Diagnose Torsionsdystonie gestellt; wohl hauptsächlich wegen des eigenartigen, an diese Krankheit erinnernden Ganges und des Wechsels von $\mathrm{Hypotonie}$ und Hypertonie in den einzelnen Muskelgruppen. Nach 2 Monaten bringt ihn die Mutter wieder, da es ihm schlechter ginge. Er selbst macht über seinen Zustand folgende Angaben: Schmerzen habe er die ganze Zeit iber keine gehabt, nur die Hände seien ihm immer kalt. Mit dem Gehen und Schreiben sei es noch schlechter geworden als früher. Auch das Sprechen sei nicht richtig. Nähere Angaben über diese Störungen kann er nicht machen. Beim Essen verschlucke or sich häufig. Lachen müsse er ebenso viel wie früher, es lache immer von selbst. Wenn der Vater ihn haue, weine er und gleich hinterher müsse er lachen.

Befund vom 29. und 30. II. 1920 und den folgenden Tagen: Sehr dürftiger Allgemeinzustand. Hände, Füße und Unterschenkel cyanotisch, hält den Mund dauernd geöffnet, lacht fast ständig unmotiviert vor sich hin, bricht, wenn man ihn anredet, zuweilen in lautes Lachen aus, weint aber auch gelegentlich unmotiviert. Das Lachen macht den Eindruck des Zwangsmäßigen. Vermehrte Salivation, läßt zuweilen etwas Speichel über die Lippen laufen. Auf Anreden gelegentlich Neigung zum Grimassieren. Urin: E.-Z.-Ug. —. Die Pupillen sind mittelweit, die 1. nicht ganz rund; beide Reaktionen prompt. Am Rande der Cornea sieht man beiderseits einen dunklen, braungrünlichen Streifen. Differenz in der Mundinnervation: der $r$. Mundwinkel wird meistens etwas stärker innerviert und nach oben gezogen, während der l. nach unten abweicht. Die Zunge ist auffallend groß und breit, wird gerade herausgestreckt, zittert. Die Sprache ist undeutlich, verlangsamt, gelegentlich leichtes Anstoßen, verschluckt Endsilben, lispelt. Das Schreiben ist durch Ungeschicklichkeit und die später zu beschreibenden Bewegungen unmöglich ( $\nabla g l$. Abb. 3).

Sehnenrefl. sehr lebhaft, Fußklonus wechselnd, ungemein schnell, mehr ein dauerndes Zittern als ein eigentlicher Klonus. Auffällig ist, daß er mit den Händen häufig nach den Genitalien faßt bzw. sie mit den Händen verdeckt, ohne daß man den Eindruck einer besonderen Schamhaftigkeit erhält, fast könnte man denken, daß es sich um eine gewisse Reflexbewegung handelt. Eigenartige ständige Unruhe in den Armen and Beinen, stärker in den Armen. Die Bewegungen sind zwecklos, grotesk, dauernd wechselnd, dabei in gewissem Sinne stereotyp, schlangen- und wurmförmig. Sie stellen eine eigenartige Mischung von choreiformen, athetoiden und ticartigen Störungen dar, jedoch tritt immer wieder eine gewisse Neigung zum Torquieren zutage. Auch die Wirbelsäule ist einem ständigen Lagewechsel unterworfen. In der mittleren Brustwirbelsäule wird eine lordotische Stellung bevorzugt, gelegentlich kommt es zu skoliotischen Verkrümmungen nach den Seiten, die ruckartig erfolgen. Das Gesicht beteiligt sich an allen diesen Bewegungen nicht. Beim Stehen sind die Bewegungen am lebhaftesten. Steht breitbeinig, die Fiiße meistens nach innen rotiert, eigentümlich verdreht, zieht bald die r., bald die l. Schulter hoch, dreht den Oberkörper bald nach r., bald nach 1., neigt ihn bald nach einer, bald nach der anderen Seite, gelegentlich stoßweise, fast schleudernd, hält Hände und Arme meist eng an den Körper gepreßt, dreht jedoch auch diese bald nach innen, bald nach außen. Beim Gehen hält er die Arme entweder krampfhaft an die Seite gepreßt, schlingt die Hände ineinander oder legt sie über den Rücken. Geht mit eigentümlich kleinen trippelnden, tänzelnden 
Schritten, die Beine stark nach vorn seitlich schleudernd, ein Bein vor das andere setzend, mit den Hacken den Boden stampfend, ein Mittelding zwischen Ataxie und hysterieformer Bewegungsstörung. Die Schultern werden beim Gehen hin und her geschleudert, soda B der Körper ständig Drehbewegungen ausführt. Unterbrochen wird das Gehen oft durch ein sprunghaftes Hüpfen nach der Seite oder nach vorn. So bietet der Gang ein ständig wechselndes Bild. Die relative Hastigkeit, mit der er sich bewegt, steht im Widerspruch zu seiner großen Ungeschicklichkeit. Rückwärtsgehen kann er kaum, dabei kleben die Füße gewissermaßen am Boden; er wagt nicht, einen Fuß hochzuheben, stürzt beim Versuch hin.

Auch im Liegen nimmt Pat, eine eigenartige, verdrehte Haltung ein.

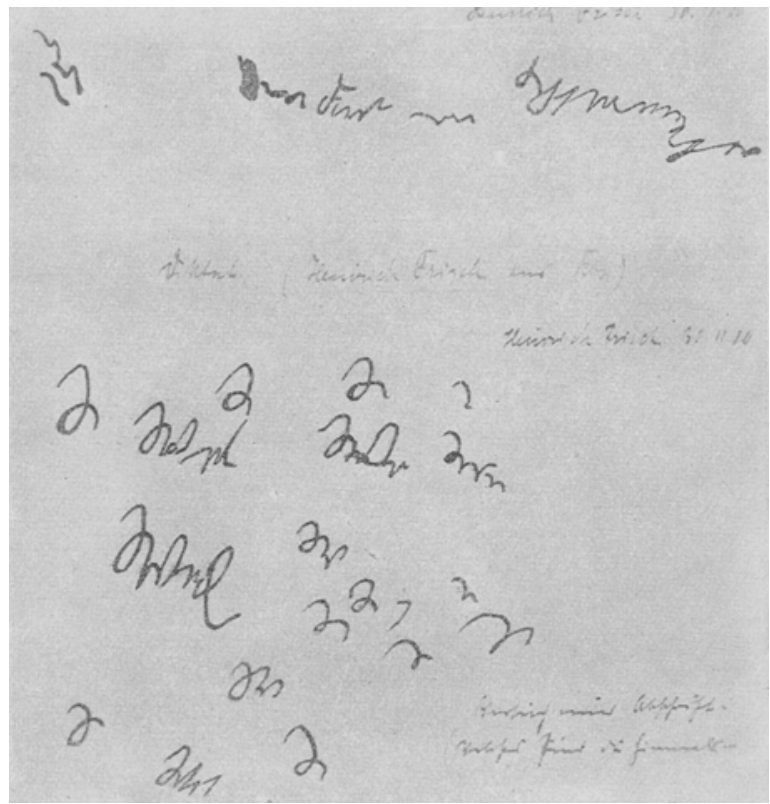

Abb. 8.

Bei passiven Bewegungsversuchen fällt der dauernde Wechsel zwischen Hypo- und Hypertonie in den einzelnen Muskelgruppen auf, besonders im 1. Arm setzen bei Bewegungsprüfung ständige, spastische Widerstände ein. Bei aktiven Bewegungen tritt in gewissem Gegensatz dazu eine ausgesprochene Hypotonie zutage, besonders in den Beinen. So kann er mit Leichtigkeit den Unterschenkel an den Oberschenkel, diesen an den Körper anpressen. Die Finger der 1. Hand mit Ausnahme des Daumens befinden sich in einer Art Krallenstellung, können im ersten $Z$ wischenglied nicht gestreckt werden. An der r. Hand zeigt nur der kleine Finger eine Andeutung von dauernder Beugestellung. Bei Versuch, die Faust zu schließen und wieder zu öffnen, werden die Bewegungen bei Wiederholung der Prüfung immer langsaner und weniger ausgiebig.

Die Füße liegen in Rückenlage dauernd in Spitzfußstellung. Sehr empfindlich gegen Schmerzreize, macht heftige Abwehrbewegungen.

Die elektrische Untersuchung ergab eine erhöhte galvanische und faradische 
Erregbarkeit der Muskeln und Nerven ohne pathologische Reaktionen. Wassermann im Blut - Blutbild: Hb. 64. Erythrocyten 4140000 . Leukocyten 9200. Polymorph. 61\%. Lymph. 17\%. Eosin. 10,5\%. Mastz. 0,5\%. Uebgz. 10,5\%. Mono. $0,5 \%$.

Bei einer Gabe von $60 \mathrm{~g}$ Traubenzucker zeigte sich nach mehreren Stunden einmal vorübergehend eine Spur Zucker im Urin. Einträufeln von Adr. hydrobr. ins Auge war ohne Effekt. Bei Injektion von 0,001 Adr. hydrobr. trat ca. 2 Minuten nach der Injektion ein kurzes Emporschnellen des Pulses von 100 auf 180 ein, wobei Pat. bei sehr blaß und etwas unruhiger wurde.

Psychisch im ganzen recht stumpf, äußert spontan keine Wünsche, keine Klagen, steht seinem Zustand ohne Einsicht gegenüber. Die Intelligenzprüfung nach Binet-Simon ergibt einen Rüekstand von mindestens $21 / 2-3$ Jahren. Er macht bei der Prüfung einen spielerisch-zerfahrenen, leicht ablenkbaren Ejndruck.

Die Mutter, welche den Pat. angeblich nur zu ibrer Erholung in die Klinik gebracht hat, nimmt ihn nach ca. 14 Tagen wieder nach Hause, um ihn am 8. II. 1921 wiederzubringen.

Pat. ist in einem sehr elenden Zustande. Der ganze Körper ist bedeckt mit einem schuppenden Ekzem. Seit Weihnachten ginge es ihm zu Hause sehr schlecht, er könne gar nicht mehr recht gehen; das Sprechen sei auch schlechter geworden. Auch habe er in der letzten Zeit häufiger Nasenbluten. Schmerzen hätte er keine; aber der $\mathrm{x}$. Arm stürbe ihm öfters ganz ab. Auch schwitze er sehr viel. Sehen und Hören könne er gut, Stuhlgang und Wasserlassen sei auch in Ordnung. Er könne aber nachts gar nicht schlafen.

Körperlicher Befund: Stark abgemagert, Gesichtshaut und Conjunctiven sehr blaß. Dauernde starke motorische Unruhe, wirft sich fortgesetzt im Bette hin und her, macht dabei ständige, zuckende Bewegungen mit den Beinen und dem Körper. Die Arme sind wesentlich ruhiger; er hält sie krampfhaft steif an den Körper gepreßt. Trotz der großen motorischen Unruhe macht Pat. einen matten, stillen Eindruck.

Der Mund steht stets weit geöffnet, beim Sprechen bricht Pat. zeitweise in zwangsartiges $\mathrm{L}_{4}$ achen aus. Die Sprache ist bedeutend schlechter als früher, ist jetzt deutlich verwaschen, mit stark nasalem Beiklang, oft kaum verständlich. Das Sprechen fällt ihm offenbar schwer, er bringt die Worte oft nur mühsam heraus. Augen: Pupillen mittelweit, nicht ganz rund, $\mathrm{R} / \mathrm{L}+, \mathrm{R} / \mathrm{C}+$, beiderseits deutlicher Skleralring. Augenbewegungen sind anscheinend frei, jedoch werden die Augen beiderseits nicht in Endstellung gebracht.

Das ganze Gesicht macht einen eigentümlich starren Eindruck. Pat. kann den Mund nicht zum Pfeifen spitzen. Der l. Mundfac. wird etwas schwächer innerviert als der r. Innere Organe o. B. Blut: Hb. 59, im Ausstrich keine pathologischen Zellen oder Zellverschiebung. Reflexe: In den oberen Extremitäten nicht sicher auslösbar, Kn./Ph. schwach, Ach./Ph. +, Abdom.-Refl. +, Ba. bds? Kein Klonus.

Liegt in eigentïmlich verdrehter zwangsartiger Haltung auf der rechten Seite, die Arme fest an den Körper gepreßt, die Beine an den Leib gezogen. Beim Versuche, die Beine zu strecken (Auslösen der Kniereflexe) schnellen sie sofort in die Höhe. Kann sich in Rückenlage nicht halten, rollt immer wieder auf die rechte Seite herüber. Der l. Arm wird im Ellenbogengelenk gestreckt, der r. gebeugt gehalten, in beiden sehr starke Spasmen, die bei wiederholten Bewegungen deutlich nachlassen. R. beimVersuch, den Arm zu strecken, deutliche Schmerzäu Berung. Rohe Kraft $r .=0$, l. Händedruck eben angedeutet. In den Armen und besonders in den Schultern ständige kleine Zuckungen. Führt größere aktive Bewegungen mit den Armen nicht aus, kann allein keine Speisen zu Munde führen, nicht zufassen. 
In den Beinen ist die Rigidität bedeutend geringer wie in den Armen und in ihrer Intensität sehr wechselnd, r. im ganzen stärker als 1 . Beim Versuch, die Beine von der Unterlage abzuheben, macht er einige unregelmäßige, ausfahrende Bewegungen bei gebeugtem Kniegelenk. Rohe Kraft $=0$.

Kann allein nicht stehen oder gehen, knickt sofort in den Knien zusammen und setzt sich auf die Erde; ist dabei außerordentlich ängstlich. Wenn unterstützt, macht er hastig einige trippelnde Schritte mit gegeneinander gebogenen Knien, einwärts gerichteten Füßen.

Kann nur breiige Speisen essen, bringt nur mit Mühe etwas herunter.

Während der nächsten Tage verfällt Pat. zunehmend. Die motorische Unruhe, die ihn nur während des. Schlafes für kurze Zeit verläßt, nimmt ständig zu. Die Sprache wird immer schlechter, Pat. stößt zuletzt fast nur noch unartikulierte Sohreie aus. Soweit eine Verständigung möglich ist, scheint Pat. fast bis in die letzten Tage hinein klar. Läßt anfangs seltener, dann immer häufiger Stuhl und Urin unter sich. Auffallend ist besonders ein in den ersten Tagen kaum zu stillendes Nasenbluten, das dann später nachläßt. Am 27. Ir. 1921 Exitus letal.

Sektionsbefund (Auszug): Leiche eines $12^{1 / 2}$ Jahre alten Knaben in schlechtem Ernährungszustand. Thymus ist vergrößert, von weicher, schlaffer Konsistenz und braunroter Farbe, sonst sind die Brustorgane ohne krankhaften Befund. Die Milz ist stark vergrößert und fühlt sich derb an. Auf dem Durchschnitt ist die Zeichnung gut sichtbar. Nieren o. B., ebenso der Magen-Darmtractus. Die Leber ist 26: 16: $8 \mathrm{~cm}$ groß und von fester Konsistenz. Ihre Oberfläche ist höckrig dadurch, daß dicht nebeneinanderliegende erbsengroße bis pfenniggroße Partien leicht konvex das Niveau überragen. Die Farbe ist graubraunrot, zwischen den einzelnen Feldern hellere Stränge. Auf dem Durchschnitt dasselbe gefelderte Bild. Leber mikroskop. Knotige Hyperplasie der Leberzellen mit auffallender Größendifferenz und herdförmiger unregelmäßiger Verfettung. Schmale Bindegewebsmäntel um die hyperplastischen Partien. Keine nennenswerten Gallengangsregenerate. Hyperämie der interstitiellen Gefäße.

Der mikroskopische Befund des Zentralnervensystems steht noch aus. Mikroskopisch war irgendeine krankhafte Veränderung nicht sichtbar.

Beide hier beschriebenen Fälle gehören ihren Symptomen, ihrem Verlaufe nach zu den Erkrankungen des extrapyramidalen Systems. Als Hauptkrankheitszeichen haben sie beide in ausgesprochenstem Maße Störungen der Muskelinnervation, wie sie Strüm pell unter dem Namen des amyostatischen Symptomenkomplexes zusammengefaßt hat.

Während der erste Fall wohl als typische Torsionsdystonie aufgefaßt werden muß, haben wir bei dem zweiten Fall lange geschwankt. Zuerst wurde auch er für eine Torsionsdystonie gehalten und auch als solche vorgestellt. Erst im weiteren Verlaufe entschieden wir uns für die Diagnose: Wilsonsche Krankheit oder Pseudosklerose ${ }^{\mathbf{1}}$ ). Wir identifizieren diese beiden Krankheitsbilder, wie es die meisten Forscher jetzt tun, und wie es auch Spielmeyer ${ }^{2}$ ) auf Grund seiner letzten pathologisch-anatomischen Untersuchungen für berechtigt hält. Für eine

1) Literatur bei v. Dziembowski, Zeitschr. f. d. ges. Neurol. u. Psychiatr. Orig. 5r; spätere Literatur bei Kastan, Arch. f. Psychiatr. u. Nervenkrankheiten 60, Heft $2 / 3$.

2) Zeitsebr. f. d. ges. Neurol. u. Psychiatr., Orig. 1920, Bd. 57. 
Torsionsdystonie sprach zuerst vor allem der eigentümliche Gang mit den Verdrehungen des Körpers, ferner der Wechsel von Hypo- und Hypertonie in den einzelnen Muskelgruppen und die mehr arhythmische Art der unwillkürlichen Bewegungen von ticartigem, choreatischem, athetotischem Charakter. Gegen die Diagnose Wilsonsche Krankheit sprach das Fehlen des rhythmischen Tremors, das grobe Wackeln des ganzen Körpers, das für die Pseudosklerose so charakteristisch ist und das Fehlen der dadurch bedingten Gangstörung, die mehr an die der multiplen Sklerose erinnert. Es fehlte ihm schließlich bei Beginn der Erkrankung die stetig zunehmende Rigidität in den einzelnen Gliedmaßen, die bis zur Contracturbildung oder bis zum Auftreten bestimmter Zwangshaltungen fortschreiten kann, eine Erscheinung, die wị bei unserem Patienten erst im Endstadium der Erkrankung sahen. Viele andere Krankheitszeichen jedoch, so die schon bei Beginn des Leidens beobachtete eigentümliche Maskenstarre des Gesichts, die Dysarthrie und Dysphagie, das Zwangslachen, die Parese der Muskulatur ohne eigentliche Lähmungserscheinungen, die Veränderungen der Psyche, der Cornealring in Zusammenhang mit der Leberveränderung und endlich der schnelle letale Verlauf sprechen wohl mebr für eine Pseudosklerose. Eine Entscheidung wird vielleicht erst die mikroskopische Untersuchung bringen.

Bemerkenswert ist dieser Fall vor allem dadurch, daß er von beiden Erkrankungen, sowohl von der Torsionsdystonie als auch von der Pseudosklerose charakteristische Symptome zeigte. Wie andere in dieses Gebiet gehörige Fälle, die nicht mit Sicherheit einer der bisher aufgestellten Krankheitsgruppen einzureihen sind, spricht auch er dafür, daß es fließende Übergänge gibt und daß es mindestens heute noch verfrüht erscheint, einzelne Krankheitsbilder scharf abgrenzen zu wollen. Sie alle sind, ich folge hier den überzeugenden Ausführungen Strümpellsil), Ausdruck der gestörten myostatischen Innervation, deren verschiedene Lokalisation im Gehirn (die zum Teil noch nicht bekannt, aber wohl stets extrapyramidal gelegen) ihre verschiedenen Symptome und Verlaufsarten bedingt.

1) Neurol. Zentralbl. 1920, Nr. I. 